\title{
Religiöser Wandel als Herausforderung an die deutsche politische Kultur - Religiöse Pluralisierung und Säkularisierung als Auslöser einer (neuen) Religionspolitik?
}

\section{Einleitung - Religiöser Wandel, Religionspolitik und politische Kultur}

In den letzten Jahren hatte eine Vielzahl an öffentlichen Debatten das Spannungsverhältnis zwischen gesellschaftlicher Integration und der Zunahme religiöser Pluralität zum Gegenstand. Häufig wurde in ihnen die Überzeugung geäußert, dass in der religiösen Pluralisierung ein beachtlicher Zündstoff für den zukünftigen Zusammenhalt der deutschen politischen Gemeinschaft liege. ${ }^{1}$ Als Hauptprobleme für die aufkommenden (kulturellen) Konflikte ${ }^{2}$ wurden, je nach normativer Position, eine mangelnde Integrationsbereitschaft auf der Seite der neu zur politischen Gemeinschaft hinzugestoßenen Bürger oder eine mangelnde Aufnahmebereitschaft auf der Seite der Integrationsgesellschaft ausgemacht. ${ }^{3}$ Aus Perspektive der Steuerung politischer Systeme ist daran interessant, dass nicht mehr »die Immigranten « oder »die Gastarbeiter « im Fokus der Ablehnung stehen, sondern sich die beobachteten Haltungen allem Anschein nach gezielt auf Mitglieder muslimischer Glaubensgemeinschaften - und damit auf Angehörige einer religiösen Gemeinschaft - beziehen. ${ }^{4}$

1 Siehe besonders plakativ und provokativ: Thilo Sarrazin, Deutschland schafft sich ab. Wie wir unser Land aufs Spiel setzen, Berlin 2010.

2 Wobei an dieser Stelle gerne an die von Samuel P. Huntington (1996) in die politikwissenschaftliche Diskussion eingebrachte Rhetorik des »Kampfes der Kulturen« angeschlossen wurde. Vgl. Samuel P. Huntington, Kampf der Kulturen. Die Nengestaltung der Weltpolitik im 21. Jahrbundert, Wien 1996.

3 Gert Pickel, »Bedrohungsgefühle versus vertrauensbildende Kontakte - Religiöser Pluralismus, religiöses Sozialkapital und soziokulturelle Integration « in: Detlef Pollack / Ingrid Tucci / HansGeorg Ziebertz (Hg.), Religiöser Pluralismus im Fokus quantitativer Religionsforschung, Wiesbaden 2012, S. 228-231. Auch: Dieter Oberndörfer, »Die Abschottung der Republik: Integration statt Zuwanderung « in: Thorsten Gerald Schneiders (Hg.), Islamfeindlichkeit. Wenn die Grenzen der Kritik verscbwimmen, Wiesbaden 2010, S. 150-166; Heiner Bielefeldt, »Das Islambild in Deutschland. Zum öffentlichen Umgang mit der Angst vor dem Islam《 in: Thorsten Gerald Schneiders (Hg.), Islamfeindlichkeit. Wenn die Grenzen der Kritik verschwimmen, Wiesbaden 2010, S. 167-200.

4 Levent Tezcan, »Konzeptionelle Überlegungen zur Gegenwartsgeschichte des Verhältnisses zwischen Christentum und Islam in Deutschland « in: Gert Pickel / Kornelia Sammet (Hg.), Zwanzig Jahre nach dem Umbruch - Religion und Religiosität im vereinigten Deutschland 1989-2010, Wiesbaden 2011, S. 293-309; Alexander Yendell / Nils Friedrich, "Wahrnehmung und Akzeptanz des Islam und anderer Religionsgemeinschaften in ausgewählten europäischen 
Nicht nur deswegen stellt sich die Frage, ob es zur Bearbeitung der sich auf dem Gebiet der Religion scheinbar formierenden Probleme ein eigenständiges Politikfeld, das der Religionspolitik, bedarf.Zumindest hat es den Anschein, dass eine alleinige Beschäftigung mit den Folgen der religiösen Pluralisierung im Spektrum von Migrations- und Immigrationspolitik nicht mehr ausreichend ist, um den skizzierten gesellschaftlichen Problemen adäquat gerecht werden zu können. Relativ eindeutig werden die Adressaten der Skepsis in der deutschen Bevölkerung mittlerweile religiös identifiziert. Es sind die Muslime - und nicht mehr die diffuse Gruppe der »Ein- oder Zuwanderer «. Ob derjenige, welcher dann Ziel dieses Misstrauens wird, zugewandert ist oder bereits in der zweiten oder dritten Generation in Deutschland lebt, ist für diese Einschätzung nur begrenzt von Relevanz. Entscheidend ist die (zugeschriebene) Zugehörigkeit zu einer religiösen "Fremdgruppe«, die aus Sicht der Skeptiker eine Bedrohung für die eigene politische Gemeinschaft darstellt.

Doch nicht nur die religiöse Pluralität im Sinne eines zunehmenden Nebeneinanders unterschiedlicher Religionen scheint die Ausbildung einer eigenständigen Religionspolitik zu erfordern, oder wenn es eine solche schon geben sollte, diese vor neue Anforderungen zu stellen. Stärker noch als die Zahl der Angehörigen einer islamischen Glaubensgemeinschaft wächst in den letzten Jahrzehnten der Anteil der Konfessionslosen. Ist dieser Prozess an sich in westlichen Ländern, die seit der Aufklärung an vielen Stellen fast selbstverständlich auf eine Trennung zwischen Politik und Religion abstellen (und sich mit Bezug hierauf oft als säkular verstehen ${ }^{5}$ ) ein zuerst einmal begrenztes Problem, so wird diese Entwicklung dort prekär, wo die Interessen politisch agierender Organisationen ins Spiel kommen. Dies ist auf diesem Feld in den letzten Jahren verstärkt der Fall. So treten Organisationen ins Scheinwerferlicht, die sich als institutionalisierte Vertreter der Konfessionslosen deklarieren und darauf begründet - teils weitreichende politische und rechtliche Forderungen stellen. Exemplarisch sei hier nur der Humanis-

Ländern« in: Detlef Pollack / Ingrid Tucci / Hans-Georg Ziebertz (Hg.), Religiöser Pluralismus im Fokus quantitativer Religionsforschung, Wiesbaden 2012, S. 228-231; Detlef Pollack / Olaf Müller / Gergely Rosta / Nils Friedrich / Alexander Yendell (Hg.), Grenzen der Toleranz. Wabrnehmung und Akzeptanz religiöser Vielfalt in Europa, Wiesbaden 2014; Dorothee de Neve, »Islamophobie in Deutschland und Europa « in: Gert Pickel / Oliver Hidalgo (Hg.), Religion und Politik im vereinigten Deutschland. Was bleibt von der Rückkehr des Religiösen? Wiesbaden 2013, S. 195-220; Gert Pickel, »Bedrohungsgefühle versus vertrauensbildende Kontakte - Religiöser Pluralismus, religiöses Sozialkapital und soziokulturelle Integration «, aaO. (FN 3), S. 246-250.

5 Wobei dies ein relativ enges Verständnis von Säkularität darstellt, da es allein auf einen Teilprozess der funktionalen Differenzierung - den zwischen Kirche und Staat - abhebt. Moderne Betrachtungen der Religionssoziologie würden mehrere Dimensionen von Säkularität als möglich und untersuchenswert erachten. Vgl. z.B. Gert Pickel, »Die Situation der Religion in Deutschland - Rückkehr des Religiösen oder voranschreitende Säkularisierung? « in: Gert Pickel / Oliver Hidalgo (Hg.), Religion und Politik im vereinigten Deutschland. Was bleibt von der Rückkehr des Religiösen?, Wiesbaden 2013, S. 65-102. 
tische Verband Deutschlands (HVD) genannt. ${ }^{6}$ Diese Verbände und ihre Vertreter wollen, neben der Abschaffung von Staatsleistungen und anderen Veränderungen in der rechtlichen Stellung von Religions- und Weltanschauungsgemeinschaften, vor allem eine den Religionsgemeinschaften gleichwertige politische wie finanzielle Anerkennung.

Auf solche konkrete Anfragen an die Politik kann diese praktisch und pragmatisch reagieren - wenn ihr die politischen Konsequenzen (z.B. Wahlverhalten) relevant und das öffentliche Interesse hoch genug erscheinen. Die Forderungen werden hinsichtlich der dahinter stehenden Gruppengrößen analysiert, kanalisiert und damit aber auch der Kritik viel an ihrer Schärfe genommen. Einfach gesagt: die Forderungen werden ganz normal im politischen Prozess verarbeitet. Für das Zusammenleben in einem politischen System gefährlicher wird es, wenn die Heterogenität der Gesellschaft zu größeren Diskrepanzen auf der Gemeinschaftsebene der politischen Kultur führt. So ist man sich in der politischen Kulturforschung weitgehend einig, dass jede politische Gemeinschaft und damit jede politische Ordnung eine allgemeine Ebene des Zusammenhalts und der Gemeinsamkeit benötigt, um nicht irgendwann auseinanderzufallen. ${ }^{7}$ Wird die gemeinsame Wertegrundlage eines politischen Systems auf die Probe gestellt, dann ist die politische Struktur selbst in Gefahr. In den Diskursen der Integrationsdebatte kommt exakt diese Ebene der politischen Kultur in den Blick und in die Diskussion, wird doch gerade hier oft das Szenario eines Auseinanderdriftens der (deutschen) Gesellschaft gezeichnet.

Vor dem Hintergrund dieser Überlegungen werden in dem vorliegenden Beitrag folgende Fragestellungen untersucht: (1) Gibt es einen Wandel der religiösen Situation in Deutschland? (2) Führt dieser zu einer Desintegration der Gesellschaft im Sinne einer Herausforderung der politischen Kultur? (3) Erlangt Religion dadurch einen neuen politischen Stellenwert und ist möglicherweise eine (neue) Religionspolitik vonnöten, um dieser Herausforderung zu begegnen? Auf diese Fragen soll anhand der Betrachtung und Analyse aktuellen empirischen Materials Antwort gegeben werden. ${ }^{8}$ Ziel ist es dabei insbesondere die sich etablierende Herausforderungsstruktur an die Politik aufgrund des religiösen Wandels herauszuarbeiten. Nicht im Fokus des Aufsatzes steht, die bisherigen konkreten Reaktionen der Religionspolitik zu analysieren und zu diskutieren. ${ }^{9}$

6 Vgl. Anja Gladkich / Gert Pickel, »Politischer Atheismus. Der neue Atheismus als politisches Projekt oder Abbild empirischer Realität? « in: Gert Pickel / Oliver Hidalgo (Hg.), Religion und Politik im vereinigten Deutschland. Was bleibt von der Rückkehr des Religiösen?, Wiesbaden 2013, S. 125-148.

7 Hier handelt es sich um die Prämissen der politischen Kulturforschung, die im Abschnitt 2 explizit vorgestellt werden.

8 Hierbei werden verschiedene Umfragedatensätze verwendet. Besonders interessant sind dabei die aktuellen Daten des Bertelsmann Religionsmonitors 2013 und der Allgemeinen Bevölkerungsumfrage der Sozialwissenschaften 2012. Für die Möglichkeit der Auswertung des Bertelsmann Religionsmonitors danke ich ausdrücklich der Bertelsmann-Stiftung. Für die Bereitstellung der verschiedenen anderen Daten der GESIS in Köln. Keine der beiden Institutionen trägt irgendeine Verantwortung für die hier präsentierten Ergebnisse. Für hilfreiche Kommentare danke ich Frau Larissa Zücker sowie meinem Kollegen Antonius Liedhegener.

9 Vgl. die Beiträge von Antonius Liedhegener und Frank Schenker im vorliegenden Heft. 


\section{Politische Kultur, politische Kulturforschung und religiöse Kulturen}

Will man die Wirkung der religiösen Pluralisierung auf die politische Kultur betrachten, dann ist es notwendig, einige Schlüsselkonzepte der politischen Kulturforschung kurz in den Blick zu nehmen. Veränderungen auf der strukturellen Ebene der Religion wirken sich selten unmittelbar auf die politische Kultur aus, vielmehr bedarf es einer Vermittlung der religiösen Veränderungen über Einstellungen und Werthaltungen der Bürger. So beeinflussen religiöse Einstellungen und persönliche Haltungen zur Religion - und weniger die reine Zugehörigkeit zu einer religiösen Organisation - politische Einstellungen. Eine sichtbare Wirkung auf der Ebene der politischen Struktur (aufgrund politischer Maßnahmen oder Institutionalisierungen von Behörden und politischen Organisationseinheiten) erfolgt dann über die eher diffusen Beziehungen zwischen politischer Kultur und politischer Struktur. So wirken religiöse Einstellungen nur dann auf die politische Struktur, wenn es ihnen gelingt die politische Kultur in einzelnen Punkten nachhaltig zu verändern. ${ }^{10}$ Sie müssen also für das politische Einstellungsgefüge der Bürger eine Relevanz besitzen und nicht als politisch irrelevant angesehen werden. Zudem benötigt eine entsprechende gesellschaftliche und politische Wirksamkeit sowohl die Bündelung individueller Einstellungen als auch das Interesse größerer Gruppen der Bevölkerung. ${ }^{11}$ Damit ist man bei der Frage nach der Definition dessen, was politische Kultur eigentlich ist. ${ }^{12}$

In dem diesem Aufsatz zugrunde liegenden Verständnis handelt es sich bei politischer Kultur um die gesammelten Einstellungen eines Kollektivs gegenüber politischen Objekten. ${ }^{13}$ Diese Sichtweise entspricht der klassischen politischen Kulturforschung und schließt an die Überlegungen von Almond und Verba ${ }^{14}$ genauso an wie an die strukturfunktionalistischen Arbeiten Talcott Parsons. ${ }^{15}$ Die zentrale Annahme der politischen

10 Sie können zum Beispiel das Wahlverhalten strukturieren. Dies gelingt aber nur, wenn religiöse oder mit Religion verbundene Einstellungen eine solche Bedeutung für das Individuum besitzen, dass sie sich gegenüber anderen Präferenzen (wirtschaftliche Lage, Umweltschutz, etc.) durchsetzen können.

11 Vgl. Antonius Liedhegener, Macht und Einfluss von Religionen. Theoretische Grundlagen und empirische Befunde der politischen Systemlehre und politischen Kulturforschung, in: Antonius Liedhegener / Andreas Tunger-Zanetti / Stephan Wirz (Hg.), Religion - Wirtschaft - Politik. Forschungszugänge zu einem aktuellen transdisziplinären Feld (= Religion - Wirtschaft - Politik, Bd.1) Baden-Baden - Zürich_2011, S. 241-273.

12 Dies ist keine unwesentliche Frage, wurde doch noch in den 1980er-Jahren hier einiges an Defiziten ausgemacht. Siehe hierzu exemplarisch Max Kaase, »Sinn oder Unsinn des Konzeptes 〈Politische Kultur〉 für die vergleichende Politikforschung, oder auch: Der Versuch einen Pudding an die Wand zu nageln « in: Max Kaase / Hans-Dieter Klingemann (Hg.), Wablen und politisches System - Analysen aus Anlass der Bundestagswabl 1980, Opladen 1983, S. 144-171.

13 Susanne Pickel / Gert Pickel, Einführung in die vergleichende politische Kultur- und Demokratieforschung, Wiesbaden 2006, S. 49-57.

14 Vgl. Gabriel Almond / Sidney Verba, The Civic Culture. Political Attitudes and Democracy in Five Nations. Princeton 1963.

15 Aus pragmatischen Gründen wird auf Erweiterungen des politischen Kulturansatzes, zum Beispiel über Symboliken und einer stärkeren Betonung der Deutungskultur gegenüber der Sozialkultur, verzichtet. Vgl. Karl Rohe, »Politische Kultur und ihre Analyse. Probleme und Perspektiven der politischen Kulturforschung « in: Historische Zeitschrift, Band 250 (1990), S. 321-346.; Talcott Parsons, The Social System, New York 1951. 
Kulturforschung ist, dass nur dann, wenn eine Korrespondenz zwischen politischer Struktur und politischer Kultur besteht, ein politisches System langfristig und zukünftig stabil sein kann. Die Konzentration auf politische Einstellungen systematisiert die Begriffsverwendung und ermöglicht empirische Untersuchungen. ${ }^{16}$ David Easton differenzierte das Konzept und identifizierte die Zielpunkte der politischen Einstellungen der Bürger und die Form der Beziehung zwischen den Bürgern und dem politischen System. Diese Beziehungen bezeichnete er als politische Unterstützung. Sie ist auf drei zentrale Objekte des politischen Systems gerichtet: Die politische Gemeinschaft, das politische Regime und die politischen Autoritäten. ${ }^{17}$ Die Unterstützung des politischen Regimes reflektiert die Haltung der Bürger zu den Kerninstitutionen des politischen Systems und die Bewertung ihrer Rollen (also beispielsweise die Position des Parlaments im politischen System). Bei den politischen Herrschaftsträgern richtet sich der Blick der Bürger auf die konkreten Inhaber politischer Autoritätsrollen. Profan gesagt, es werden Aussagen über die Politiker als Personen oder aber auch als Sammelgruppierung getroffen. Die für die vorliegende Fragestellung der Wirkung von Religion besonders interessante politische Gemeinschaft umfasst die Einstellungen der Mitglieder eines politischen Systems gegenüber einer übergeordneten Gemeinschaft und ihre grundlegenden Wertmuster. Gemeinschaftssinn und eine übergreifende Objektzuordnung (wie z.B. die Nation und die in ihr lebenden Personen) sind die Basisprinzipien dieser Komponente der politischen Ordnung, die sich in Zugehörigkeitsgefühl zu dem Kollektiv und einer gegenseitigen Loyalität der Gemeinschaftsmitglieder äußert.

Die bewertenden Orientierungen resultieren aus unterschiedlichen Quellen. Eine Quelle ist die Zufriedenheit mit den Outputs des politischen Systems bzw. mit den Autoritäten, die diese Outputs produzieren. Easton nennt sie spezifische Unterstützung (specific support). Sie besitzt einen konkret - teilweise personell - fassbaren Bezugspunkt der entsprechenden politischen Einstellungen in der Realität, ist den politischen Herrschaftsträgern zugewiesen und lebt aus der Wahrnehmung der Performanz der politischen Herrschaftsträger und der Zufriedenheit der Bürger mit deren Leistungen. Davon $\mathrm{zu}$ unterscheiden ist die diffuse Unterstützung (diffuse support): Das Objekt wird nicht unterstützt, weil es bestimmte Leistungen erbringt, sondern um »seiner selbst willen«. Diese findet sich vor allem auf der Ebene der Unterstützung der politischen Gemeinschaft (Abb. 1).

16 Dabei soll nicht verschwiegen werden, dass es weitreichende Diskussionen gerade über Verständnis und Definition von politischer Kultur gab. Zumeist zielten sie auf die immer noch zu geringe Präzision der Begriffsfassung. Immerhin besitzt sie den Vorzug, dass dadurch sehr diffuse Verwendungen von politischer Kultur im Sinne von Politikstilen, Umgang unter politischen Gegnern oder noch allgemeiner gefasst als eher unsystematische Bezeichnungen ausgeschlossen werden und ein analytischer Zugang beansprucht werden kann. Vgl. auch Pickel / Pickel, Politische Kultur- und Demokratieforschung, aaO. (FN 13), S. 58.

17 Vgl. David Easton, A System Analysis of Poltical Life, New York 1979; David Easton, »A ReAssessment of the Concept of Political Support « in: British Journal of Political Science 5, 1975, S. 435-457. 
Abb. 1: Das Konzept politischer Unterstützung nach David Easton

\section{Unterstützungsobjekte}

Politische Regime

Autoritäten

Gemeinschaft

Diffus

Art der Unterstützung

Spezifisch

Quelle: Fucbs1989: $18 .^{18}$

\begin{tabular}{|c|c|c|}
\hline $\begin{array}{c}\text { Identifikation } \\
\text { mit der } \\
\text { politischen } \\
\text { Gemeinschaft }\end{array}$ & $\begin{array}{c}\text { Regime- } \\
\text { Legitimität }\end{array}$ & $\begin{array}{c}\text { Autoritäten- } \\
\text { Legitimität }\end{array}$ \\
\cline { 2 - 3 } & $\begin{array}{c}\text { Regime- } \\
\text { Vertrauen }\end{array}$ & $\begin{array}{c}\text { Autoritäten- } \\
\text { Vertrauen }\end{array}$ \\
\hline & & $\begin{array}{c}\text { Zufriedenheit mit den } \\
\text { alltäglichen Outputs }\end{array}$ \\
\hline
\end{tabular}

Ein zentraler Bezugspunkt der politischen Kulturforschung und des Ansatzes der politischen Unterstützung ist der Gedanke der Integration. Seitens der politischen Kulturforschung wird Integration vor allem als politische Unterstützung (im Sinne von Einstellungen der Bürger) auf der Ebene der politischen Gemeinschaft verstanden. Man steht einer übergeordneten politischen Gemeinschaft nicht grundsätzlich ablehnend gegenüber und erkennt ihre grundsätzlichen Prinzipien an. Für die vorliegende Fragestellung bedeutet dies, dass in einer Migrationsgesellschaft nicht eine größtmögliche kulturelle Assimilation der Minderheiten an die Mehrheit, sondern allein eine gemeinsame Anerkennung der für den Erhalt des politischen Systems wichtigen Grundprämissen und Werte das Kriterium für eine gelungene Integration ist. Für Demokratien sind dies die Anerkennung der Demokratie als angemessenster Herrschaftsordnung und ein allgemeines Zugehörigkeitsgefühl zur nationalen politischen Gemeinschaft. ${ }^{19}$

Die Wirkung von Religion und religiöser Pluralisierung ist nicht auf diese Ebene der diffusen politischen Unterstützung (und die politische Gemeinschaft) beschränkt. Politische Entscheidungen, welche in Reaktion auf Forderungen von Religionsgemeinschaften seitens der politisch Verantwortlichen getroffen werden müssen, können die Ebene der politischen Unterstützung von Autoritäten - und darüber vermittelt des politischen Systems - erreichen. Dies betrifft Entscheidungen, welche seitens der politisch Verantwortlichen hinsichtlich religiös konnotierter Probleme getroffen werden und wie die erfolgte Problemlösung seitens der Bürger dann beurteilt wird. Allerdings stehen diese Einzelentscheidungen immer neben einer Vielzahl anderer Einzelentscheidungen. Entsprechend ist ihre Wirkung für die politischen Grundhaltungen der Bürger begrenzt.

18 Dieter Fuchs, Die Unterstützung des politischen Systems der Bundesrepublik Deutschland, Wiesbaden 1989, S. 18.

19 In gewisser Weise hat sich dabei in Demokratien die Interpretation des Objektes »politische Gemeinschaft « etwas von der Gemeinschaft weg hin zu demokratischen Grundprämissen verschoben. Siehe hierzu Dieter Fuchs, »Das Konzept der politischen Kultur: Die Fortsetzung einer Kontroverse in konstruktiver Absicht « in: Dieter Fuchs / Edeltraut Roller (Hg.), Bürger und Demokratie in Ost und West: Studien zur politischen Kultur und zum politischen Prozess, Wiesbaden 2002, S. 27-49. 
Diskrepanzen auf der Ebene der politischen Gemeinschaft sind da weit gefährlicher einzuschätzen, sind doch einerseits die Beziehungen hier diffus und schwieriger ermittelbar wie auch schwieriger beeinflussbar, andererseits wird die allgemeinste Ebene des $\mathrm{Zu}$ sammenhalts eines politischen Regimes auf die Probe gestellt. ${ }^{20}$ Wie können diese Prämissen der politischen Kulturforschung nun mit dem Feld einer Religionspolitik in Verbindung gesetzt werden? Eastons Konzept enthält nicht nur die Unterstützung (support). Um diesen zu erlangen benötigen die politischen Regime auch An- oder Herausforderungen. Diese Seite bezeichnet Easton als demand. Auf die politischen demands sind dann politische Reaktionen notwendig um support zu erhalten. Können nun religiöse Überzeugungen und Zugehörigkeiten demands entwickeln, welche die politische Kultur belasten können? Dazu ist es angebracht einen Blick auf die Entwicklung des Religiösen in Deutschland zu werfen und zu fragen, inwieweit sie entsprechende $d e-$ mands produziert.

\section{Religiöser Wandel: Säkularisierung und Pluralisierung}

In den letzten Jahrzehnten hat in Deutschland auf dem religiösen Sektor ein tiefgreifender Wandel stattgefunden. An erster Stelle ist auf den kontinuierlichen Säkularisierungsprozess zu verweisen, der vermutlich bereits in den 1950-Jahren einen Anfang nahm, aber spätestens seit den 1970er-Jahren deutlich erkennbar wurde. Diese Entwicklung manifestiert sich in einem Traditionsabbruch christlicher Religiosität und in einer stetigen Zunahme des Anteils der Konfessionslosen in Deutschland, die bis heute anhält. ${ }^{21}$ Und dieser Verlustprozess betrifft West- wie Ostdeutschland gleichermaßen, wenn auch auf der Basis unterschiedlicher Ausgangsbedingungen. So findet sich in Ostdeutschland bereits eine »Kultur der Konfessionslosigkeit«, während wir in Westdeutschland - allen Abbrüchen zum Trotz - noch eine weitverbreitete »Kultur der Konfessionsmitgliedschaft« haben. ${ }^{22}$ Hierfür sind zweifelsohne die gesellschaftspolitischen Entwicklungen vor 1989 verantwortlich. Kam es in der ehemaligen DDR zu einer massiven, und durch den Staat forcierten, Zurückdrängung von Kirche und religiösen Werten, so konnten sich

20 So wirkt auf der Ebene der politischen Gemeinschaft vornehmlich die diffuse Unterstützung. Sie zeichnet sich durch eine relativ niedrige Gerichtetheit, aber dadurch auch durch eine hohe Legitimität und Legitimationskraft aus.

21 Siehe zusammenfassend Detlef Pollack, Säkularisierung - ein moderner Mythos?, Tübingen 2003; Detlef Pollack, Rückkehr des Religiösen?, Tübingen 2009, S. 125-222; Pickel, »Die Situation der Religion in Deutschland « aaO. (FN 5), 65-102; Gert Pickel / Kornelia Sammet (Hg.), Zwanzig Jabre nach dem Umbruch - Religion und Religiosität im vereinigten Deutschland 1989-2010. Wiesbaden 2011. Antonius Liedhegener, Säkularisierung als Entkirchlichung. Trends und Konjunkturen in Deutschland von der Mitte des 19. Jahrhunderts bis zur Gegenwart, in: Karl Gabriel / Christel Gärtner / Detlef Pollack (Hg.), Umstrittene Säkularisierung. Soziologische und historische Analysen zur Differenzierung von Religion und Politik. Berlin 2012, S. 481-531.

22 Vgl. Gert Pickel, »Konfessionslose in Ost- und Westdeutschland - ähnlich oder anders? « in: Detlef Pollack / Gert Pickel (Hg.), Religiöser und kirchlicher Wandel in Ostdeutschland 1989-1999, Opladen 2000, S. 207. 
die christlichen Kirchen in Westdeutschland auf Basis von Religionsfreiheit und Staatskirchenverträgen relativ ungestört entwickeln. Der trotzdem seit den 1970er-Jahren einsetzende Prozess massiven Verlustes in der Mitgliedschaft und bei den religiösen Gemeinschaftspraktiken hatte im Kern andere Gründe als in der DDR. Modernisierungsprozesse, zunehmende Individualisierung, aber auch eine höhere innergesellschaftliche Migration und zunehmende Urbanisierung werden hier genannt.. ${ }^{23}$ Gleichzeitig wird auch auf die Bildungsexpansion und den in Teilen mit ihr verbundenen Wertewandel zu Selbstentfaltungswerten hingewiesen.

Heute ist die Parallelität der Entwicklung in West- und Ostdeutschland bemerkenswert. Hatte man noch 1989 angenommen, in den neuen Bundesländern würde es, wenn die Repression wegfiele, zu einer - sogar massiven - Rückkehrbewegung zu den christlichen Kirchen kommen oder zumindest eine größere Suche nach neuen Formen religiöser Erfahrung und Zugehörigkeit einsetzen, dann wurden beide Hoffnungen enttäuscht. Auch nach 1989 verloren die beiden christlichen Großkirchen in den neuen Bundesländern weiter an Mitgliedern. Diese Verluste drückten sich hauptsächlich in einer Zunahme von Konfessionslosen aus und nicht in einem Aufkommen vieler neuer religiöser Bewegungen bzw. einem breiteren Mitgliedschaftszuwachs solcher Gruppen. Im Einzelnen zeigt sich entgegen der noch 1989 oft geäußerten Annahme einer Anpassung der Bevölkerung im Osten Deutschlands an den Westen hinsichtlich Religiosität und Kirchlichkeit gegenwärtig vielmehr eine Angleichung Westdeutschlands an die religiöse Situation in Ostdeutschland - zumindest was die Kirchenmitgliedschaft angeht. ${ }^{24}$

Selbst wenn man hinsichtlich der in der Religionssoziologie geführten theoretischen Diskussion seine Position offen hält ${ }^{25}$, scheint zumindest die Verankerung der bislang dominierenden christlichen Großkirchen in der deutschen Bevölkerung kontinuierlich im Schwinden begriffen. ${ }^{26}$ Immer seltener sind die Deutschen Mitglied in einer der großen Volkskirchen oder überhaupt einer Religionsgemeinschaft, immer seltener werden religiöse Praktiken und Riten vollzogen, immer seltener sind traditionelle religiöse Normen bindend für die Bürger und immer seltener werden Alltagsentscheidungen aufgrund religiöser Überzeugungen getroffen.

23 Gert Pickel, Religionssoziologie. Eine Einführung in zentrale Themenbereiche, Wiesbaden 2011, S. 137-177.

24 Gert Pickel, »Religiosität versus Konfessionslosigkeit « in: Michaela Glaab / Werner Weidenfeld / Michael Weigl (Hg.), Deutsche Kontraste 1990-2010. Politik - Wirtschaft - Gesellschaft - Kultur, Frankfurt/Main 2010, S. 447-484.

25 Pickel, Religionssoziologie, aaO. (FN 23), S. 135-226.

26 Dabei geht es auch um die Frage, ob Konfessionsmitgliedschaft oder Gottesdienstbesuch ausreichende Indikatoren für einen sozialen Bedeutungsverlust von Religion darstellen, wie es die Säkularisierungstheorie postuliert. Pollack, »Säkularisierung - ein moderner Mythos? «aaO. (FN 20), S. 77-130. 


\section{Abb. 2: Die Entwicklung der Konfessionslosigkeit in Deutschland}

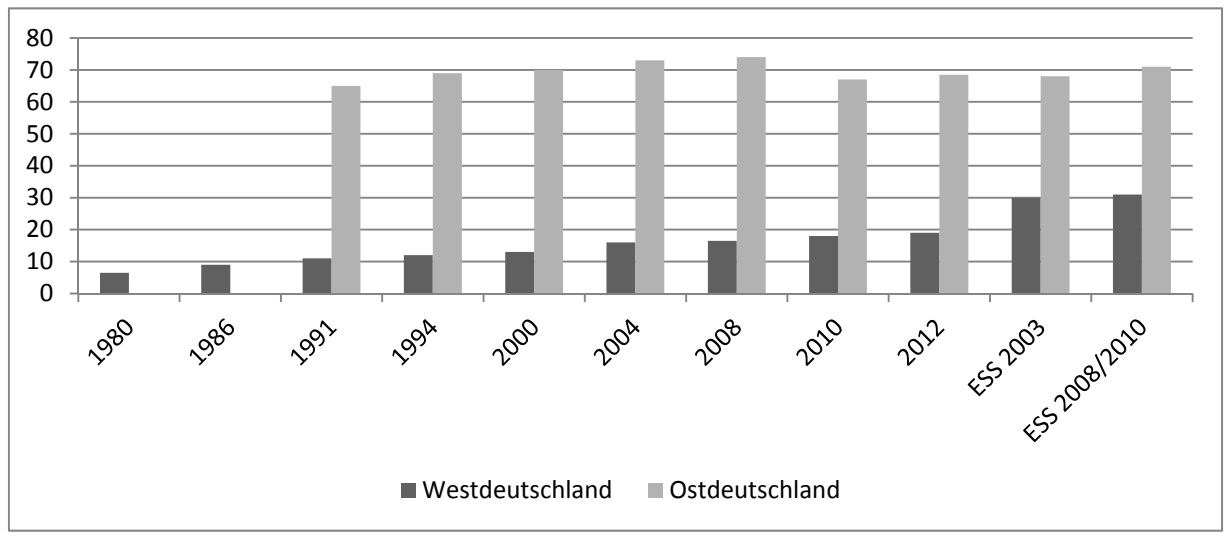

Quelle: Eigene Berechnungen auf Basis Allgemeine Bevölkerungsumfrage der Sozialwissenschaften 1980-2012; European Social Surveys 2003, 2008/2010. ${ }^{27}$

So ist auch - obwohl dies nicht impliziter Bestand der Säkularisierungstheorie ist - die Annahme plausibel, dass neben die steigende Zahl an Konfessionslosen eine steigende Zahl an sogenannten religiös Indifferenten tritt. ${ }^{28}$ Ihre Position zu Religion ist als eher uninteressiert oder »unmusikalisch - um mit Weber ${ }^{29}$ zu reden - zu beschreiben. Da trösten aus Sicht einer politischen Bedeutsamkeit von Religion Überlegungen der Individualisierungstheorie des Religiösen, die eine Transformation des Religiosen hin zur individualisierten Bastelreligiosität postulieren, nur wenig. ${ }^{30}$ Denn selbst wenn im Zuge einer Transformation des Religiösen zwar die großen Religionen an Einfluss und Bindung verlieren aber eine individualisierte Form von Religiosität verbliebe, wäre von Angehörigen dieser nur eine sehr eingeschränkte politische Wirkung zu erwarten. Eine Privatisierung des Religiösen würde dann ebenfalls einen politischen Bedeutungsverlust implizieren. Doch selbst diese Annahme eines Bedeutungsverlustes kirchlicher Institutionen bei gleichzeitigem Bedeutungsgehalt von Religiosität ist fraglich. So lässt sich über

27 Die in Abbildung 2 nicht durchgängig kontinuierlich erscheinende Entwicklung der Zunahme der Konfessionslosen ist wahrscheinlich auf eine Veränderung der Stichprobengestaltung im Allbus (Haushaltsstichprobe/Personenstichprobe) zurückzuführen. Dies führt zu einer Verschiebung der Kalkulationsbasis. Die Entwicklung geht aber auch seit 2010 in die gleiche Richtung wie in den Jahren davor, was auf eine kontinuierliche Entwicklung deutet. Dies belegen auch andere statistische Kernzahlen. Dass die Erhebungsfrage Auswirkungen auf die Eigenzuordnung haben kann, zeigen die Ergebnisse der European Social Surveys, welche für Westdeutschland höhere Zahlen an Personen ergeben, die sich als keiner Kirche zugehörig einstufen.

28 Monika Wohlrab-Sahr, »Das stabile Drittel: Religionslosigkeit in Deutschland « in: Bertelsmann Stiftung (Hg.), Woran glaubt die Welt? Analysen und Kommentare zum Religionsmonitor 2008, Gütersloh 2008, S. 151-168.

29 Max Weber, Wirtschaft und Gesellschaft. Grundriss einer verstehenden Soziologie, Tübingen, 1980.

30 Thomas Luckmann, Die unsichtbare Religion, Frankfurt/Main 1991. 
die unterschiedlichen Generationen hinweg ein kontinuierlicher Abbruch in der Bedeutungszuschreibung von Religion für das eigene Leben erkennen (Abb. 3).

Abb. 3: Wichtigkeit von Religion für das Leben

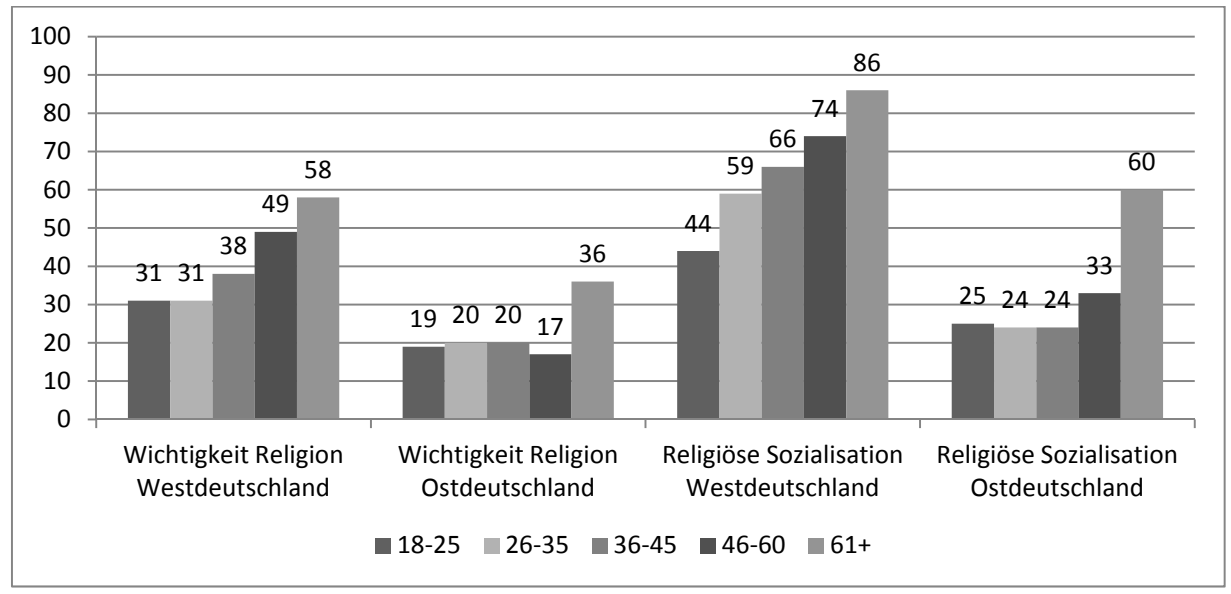

Quelle: Eigene Berechnungen auf Basis Church and Religion in an enlarged Europe 2006; Wichtigkeit Religion $=»$ Religion spielt eine wichtige Rolle in meinem Leben«; Religiöse Sozialisation $=$ »Ich wurde religiös erzogen«; zustimmende Werte zu Aussage.

Alle Überlegungen hinsichtlich einer gestiegenen öffentlichen Sichtbarkeit von Religion einmal zur Seite gestellt, scheint auf der Handlungsebene der Individuen Religion für nachwachsende Generationen nur eine begrenzte Relevanz aufzuweisen. Es scheint so als würden die sozialen und sozioökonomischen Veränderungen der Gesellschaft hier ihren Niederschlag finden und Religion aus Sicht jüngerer Gesellschaftsmitglieder immer mehr verzichtbar machen. Zentrales Verbindungsglied ist dabei die Sozialisation. Noch immer findet die religiöse Sozialisation weitgehend im Elternhaus statt, aber bereits längere Zeit wurden hier Abschmelzungen in den Sozialisationsinhalten beobachtet. Die Datenlage ist heute sehr deutlich: Wesentlich stärker noch als vor zwei Generationen scheint es massive Traditionsabbrüche in der Weitergabe von Religion zu geben. Immer weniger junge Deutsche sehen sich selbst als religiös sozialisiert an (Abb. 3). Dieser Anteil sinkt von Generation zu Generation. Mindestens genauso bedeutsam ist aber, dass damit auch die Weitergabe von religiösen Traditionen und religiösem Wissen an die eigenen Kinder erodiert. Einfach gesagt: Wenn immer weniger Menschen über eine religiöse Sozialisation verfügen (was religiöses Wissen, religiöse Erfahrungen und religiöse Praktiken sowie eine relativ klare Gottesvorstellung umfassen kann), dann können natürlich auch immer weniger Menschen eine religiöse Sozialisation weitergeben. Kommt es dann über die Generationen weiterhin zu den zusätzlichen Einbrüchen, dann dürfte der Abbruchsprozess in der Mitgliedschaft, bei den religiösen Praktiken und möglicherweise auch im Glauben in den nächsten Jahren sogar an Dramatik gewinnen. 
Doch die Säkularisierung ist nicht der einzige Prozess auf dem religiösen Sektor. Neben der Säkularisierung findet auch ein Prozess der religiösen Pluralisierung statt. ${ }^{31}$ Genau genommen könnte man auch die Zunahme der Konfessionslosen als einen Prozess religiöser Pluralisierung - oder der Pluralisierung von Weltanschauungen - verstehen. Gleichwohl wird unter religiöser Pluralisierung zumeist das Hinzutreten anderer Religionen oder der Mitglieder anderer religiöser Organisationen bzw. mit anderer religiöser Überzeugung gesehen. ${ }^{32}$ Speziell zwei Gruppen sind hier zu nennen: Zum einen ist die Zahl der Mitglieder der orthodox-christlichen Kirche in Deutschland auf immerhin 1,8 Millionen angewachsen. Dies sind vor allem Spätheimkehrer deutscher Abstammung aus dem Gebiet der ehemaligen UdSSR und ihre Kinder. Zum anderen die Bürger muslimischen Glaubens. Ihr Anteil ist mittlerweile auf einen nicht genau klassifizierbaren Anteil angewachsen, der nach offiziellen Statistiken bei 4,5 Prozent liegt, inoffiziell aber sogar höher eingeschätzt wir. Dieser Prozess ist dabei kein deutsches Sonderphänomen, sondern eine (west)europäische Generalentwicklung. Mehr und mehr wandelt sich Westeuropa zu einem multireligiösen Gebiet. Von entscheidender Bedeutung ist ein weiterführender Schluss, den man aus diesen Entwicklungen ziehen kann: Der Prozess religiöser Pluralisierung in seiner Gesamtheit wird die Entwicklung in Deutschland (und den es umgebenden europäischen Ländern) auch in den nächsten Jahren maßgeblich prägen. Oder anders gesagt: Sowohl die Zahl der Konfessionslosen als auch die Zahl der Angehörigen anderer Religionen als der beiden christlichen Großkirchen wird in $\mathrm{Zu}$ kunft weiter zunehmen.

\section{Die Folgen der Säkularisierung - Trennung von Politik und Religion?}

Doch sind aus diesen Entwicklungen weitreichende politische Folgen zu erwarten? Wenden wir uns erst einmal dem Prozess der Säkularisierung zu. Der Traditionsabbruch der christlichen Kirchen und ihr daraus resultierender Mitgliederverlust wurden bereits aufgezeigt. Da man davon ausgehen muss, dass dieser Prozess auch in der näheren $\mathrm{Zu}$ kunft weiter voranschreiten wird, entsteht ein numerischer Unterstützungsverlust der christlichen Kirchen. Anders gesagt: Eine geringere Zahl an Mitgliedern untergräbt mit der Zeit auch die Legitimationsbasis für die von ihnen ausgehenden politischen Forderungen. Soweit ist es natürlich noch nicht, ist die Mehrheit der Deutschen (anders als zum Beispiel in den benachbarten Niederlanden) doch noch Mitglied in einer Kirche. Immer häufiger aufbrechende Debatten, wie zum Beispiel hinsichtlich der Staatsleistungen für die Kirchen, deuten aber bereits in die Richtung eines Legitimitätsverlustes auf dem politischen Sektor.

31 Christoph Wolf, »Religiöse Pluralisierung in der Bundesrepublik Deutschland « in: Jürgen Friedrichs / Wolfgang Jagodzinski (Hg.), Soziale Integration. Wiesbaden 1999, S. 320-349.

32 Volkhard Krech / Markus Hero, "Die Pluralisierung des religiösen Feldes in Deutschland. Empirische Befunde und systematische Überlegungen « in: Gert Pickel/Kornelia Sammet (Hg.): Zwanzig Jabre nach dem Umbruch - Religion und Religiosität im vereinigten Deutschland 1989-2010. Wiesbaden 2011, S. 27-42. 
Doch dies ist nur eine Facette. Eine andere hängt zentral mit der Durchsetzung von Säkularisierung zusammen. Zwar wurde in den letzten Jahren verstärkt auf den Bedeutungsgewinn von Religion in öffentlichen Diskursen verwiesen. In diesem Zusammenhang ist auch das Schlagwort der »Postsäkularität « zu nennen. ${ }^{33}$ Ob diese möglicherweise häufigeren öffentlichen Diskussionen über Religion aber als eine Wiederkehr des Religiösen anzusehen sind, darf zumindest hinterfragt werden. Nehmen wir die oben dargestellten Ergebnisse ernst, so ist bei den Individuen eher ein Bedeutungsverlust von Religion für ihr Alltagsleben zu konstatieren. Aus Sicht der gesellschaftlichen und/oder politischen Bedeutsamkeit ist es dann eigentlich irrelevant, ob man im Privaten noch Religion praktiziert oder nicht, bleibt dies doch ohne weitere Auswirkung auf die Gesellschaft.

Doch es finden sich nicht nur Hinweise auf eine individuelle Säkularisierung, sondern auch Indizien für einen weitreichenden Prozess der gesellschaftlichen Säkularisierung. ${ }^{34}$ Letztere drückt die Vorstellung der Bürger aus, dass zwischen Kirche und Staat bzw. sogar zwischen Politik und Religion, eine scharfe Trennung bestehen sollte. Genau genommen ist diese Trennung auch das am häufigsten genannte Beispiel, wenn mit Bezug auf Säkularisierung von funktionaler Differenzierung gesprochen wird. Diese vollzieht sich aber eben nicht nur als faktische Trennung der Einflusssphären im Zuge von Modernisierungsprozessen, sondern hat sich bei einer überwältigenden Mehrheit der Deutschen auch in den Köpfen als Normalzustand einer modernen, demokratischen Gesellschaft festgesetzt.

Tab. 1: Das Verbältnis von Politik und Religion - funktionale Differenzierung

\begin{tabular}{l|c|c}
\cline { 2 - 3 } & WD & OD \\
\hline $\begin{array}{l}\text { Religiöse Führer sollten Regierungsentscheidungen nicht beeinflussen } \\
\text { (2006) }\end{array}$ & 70 & 70 \\
$\begin{array}{l}\text { Religiöse Führer sollten Regierungsentscheidungen nicht beeinflussen } \\
\text { (2013) }\end{array}$ & 79 & 68 \\
Die Europäische Verfassung sollte eine Referenz auf Gott beinhalten (2006) & 27 & 20 \\
$\begin{array}{l}\text { Nur Politiker, die an Gott glauben, sind für ein öffentliches Amt geeignet } \\
\text { (2013) }\end{array}$ & 9 & 11 \\
Kirche sollte politisch Stellung nehmen (2012) & 38 & 42 \\
\hline
\end{tabular}

Quelle: Eigene Berechnungen auf Basis Church and Religion in an enlarged Europe 2006; Allbus 2012; Bertelsmann Religionsmonitor 2013; WD = Westdeutschland, OD = Ostdeutschland.

So würden nicht nur die meisten West- wie auch Ostdeutschen sich eine Intervention von religiösen Führungspersönlichkeiten in die Politik in Demokratien verbieten, auch

33 Jürgen Habermas, Glauben und Wissen. Friedenspreis des Deutschen Buchbandels, Frankfurt/Main 2001; Jürgen Habermas, Zwischen Naturalismus und Religion. Philosophische Aufsätze, Frankfurt/Main 2009.

34 Eine entsprechende dimensionale Differenzierung etablierte Karel Dobbelaere, der individuelle, organisatorische und gesellschaftliche Säkularisierung voneinander unterschied. Vgl. Karel Dobbelaere, Secularization. An Analysis on Three Levels, Brüssel 2002. 
Religiosität erscheint nur einer sehr kleinen Minderheit als eine Voraussetzung für ein politisches Amt notwendig (Tab. 1). Etwas mehr - ungefähr zwei von fünf Befragten erkennen den Kirchen zumindest noch zu, dass sie zu politischen Themen Stellung nehmen sollen. Die Mehrheit macht ihnen aber noch nicht einmal dieses vergleichsweise zurückhaltende Zugeständnis. Bemerkenswert ist die Einigkeit der West- und der Ostdeutschen in diesem Punkt. Die Vorstellung, dass Religion und Kirche ein von der Politik erst einmal weitgehend getrenntes Areal zu bearbeiten haben, ist einhellig, ob man nun aus einer »Kultur der Konfessionsmitgliedschaft « oder einer »Kultur der Konfessionslosigkeit « kommt. ${ }^{35}$ Sozial darf sich Kirche engagieren, politisch aber lieber nicht.

Gesellschaftliche und speziell politische Handlungen und Entscheidungen beruhen aus Sicht der Bürger fast grundsätzlich auf rationalen - und damit zumeist säkular zu begründenden - Entscheidungen. Religiöse Argumente spielen nur in begrenzten Bereichen und Fällen eine Rolle. Dies ist Folge einer Wahrnehmung der Gesellschaft als eine weitgehend säkular geprägte und verfasste und betrifft das Gros der Alltagsentscheidungen und noch stärker politische Entscheidungen. ${ }^{36}$ Damit sei nicht die Bedeutung von Religion und Religiosität für bestimmte Wertorientierungen negiert. Haltungen zur Bioethik oder zur Sterbehilfe können - und tun dies auch - sehr wohl auf religiösen Überzeugungen beruhen. Nur werden diese Vorstellungen von den Betroffenen dann in ihre politischen Raster eingetragen - und letztendlich als politische Äußerungen verstanden. Zudem, und dies erweist sich für ihre Wirkungskraft als entscheidend, stehen sie neben anderen für das Individuum ebenfalls relevanten, wenn nicht relevanteren, Entscheidungsprinzipien.

Was ist nun aber mit der Rückkehr des Religiösen? Abgesehen von den begrenzten Indizien für diese, kann man nicht abstreiten, dass es möglich wäre, dass mehr über Religion geredet wird - und dies vor allem vor dem Hintergrund politischer Entscheidungen. Empirisch ist bislang nicht belegt, ob wirklich ein stärkerer Diskurs über und unter Einbindung von Religion besteht. ${ }^{37}$ Möglich wäre es aber immerhin. Dann hätte man es vielleicht nicht mit einer Säkularisierung auf der Ebene des öffentlichen Diskurses zu tun. Die Deutung einer Säkularisierung auf den anderen Ebenen der Gesellschaft wäre damit allerdings keineswegs widerlegt. Zumindest würden die bisherigen Säkularisierungsannahmen hinsichtlich eines Bedeutungsgewinns oder Bedeutungserhalts auf einer anderen Ebene ergänzt werden. Genau betrachtet könnte es sich bei der Ebene des öf-

35 In die gleiche Richtung eines weitreichenden Verständnisses des modernen Europa als säkular verweisen in ihrer Stärke nahezu deckungsgleiche Ergebnisse in anderen europäischen Ländern. Vgl. Gert Pickel, »Secularization as European Fate « in: Gert Pickel / Olaf Müller, Church and Religion in Contemporary Europe. Results from Empirical and Comparative Research, Wiesbaden 2009, S. 98.

36 Hier ist anzumerken, dass sich dies nicht auf die einfache optische Sichtbarkeit von Kirche bezieht. So ist Deutschland immer noch von der christlichen Kultur durchdrungen. Diese wird aber eben vielerorts immer mehr als Kultur oder Tradition, denn als religiös verbindliches Glaubenssystem wahrgenommen.

37 So sind die weiterführenden Annahmen Casanovas empirisch nur unzureichend geprüft und zudem basieren seine Annahmen generell nur auf der begrenzten Zahl von fünf Untersuchungsfällen. Vgl. Jose Casanova, Public Religions in the Modern World. Chicago 1994. 
fentlichen Diskurses weniger um einen sozialen als vielmehr um einen politischen Bedeutungserhalt handeln. So wird über Religion geredet und dabei selten wirklich religiös kommuniziert. Bislang kaum diskutierte politische und rechtliche Positionen von Religion werden vor dem Hintergrund von Säkularisierung und auch religiöser Pluralisierung in Frage gestellt oder neu diskutiert. Sozial, im Sinne der Bedeutung von Religion für das Leben des Einzelnen und die Organisation der Gesellschaft, verliert Religion dann an Bedeutung, um gleichzeitig auf der Ebene des Politischen - als potentieller Konfliktfaktor - eine spezifische neue (und möglicherweise sogar stärkere) Bedeutung zu entfalten. Diese neuen Debatten über Religion wären dann aber auch eine Frage für eine eigenständige Religionspolitik, die vielleicht immer weniger religiöse Menschen betrifft, aber soziale und politische Folgen für das Zusammenleben in der gesamten Gesellschaft verstärkt in den Blick nehmen muss.

\section{Die Folgen der religiösen Pluralisierung - Bedrobung und Integrationsprobleme}

Dieser öffentliche Debattenrahmen mit politischer Wirkung wird beim Blick auf den zweiten Prozess des Religiösen deutlich - der religiösen Pluralisierung. Sie stellt für sich gesehen als faktische sozialstrukturelle Entwicklung zuerst einmal kein größeres gesellschaftliches Problem für auf Heterogenität ausgerichtete Demokratien dar. Religionspolitisch problematisch wird sie, wenn aus ihr politische Kontroversen oder gar Konflikte entstehen. Genau dies ist der Fall bei einem Aspekt der religiösen Pluralisierung der Zunahme von Mitgliedern islamischer Glaubensgemeinschaften. Für jeden sichtbar hat sich in den letzten Jahren eine vehement geführte Debatte um die Integration von Muslimen entwickelt. In dieser treffen unterschiedliche Positionen aufeinander: Die eine Seite sieht die Probleme eindeutig auf der Seite der Muslime oder besser der Zuwanderer. Sie seien nicht bereit sich zu integrieren und etablierten eine »Parallelkultur «, wird hier behauptet. ${ }^{38}$ Normativer Hintergrund solcher Einschätzungen ist die Idee der möglichst vollständigen »Assimilation « von »Fremden «. Die andere Seite weist auf die mangelnde Gastfreundschaft des Einwanderungslandes hin und betont das durch die Religionsfreiheit in der Verfassung geschützte Nebeneinander unterschiedlicher religiöser und ethnischer Gruppen. Teilweise wird das Argument auf das Potential der, aus der neuen Vielfalt resultierenden, Bereicherung der kulturellen Landschaft des Landes erweitert. Diese Position wird in öffentlichen Diskussionen häufig als »Multikulturalismus« bezeichnet.

Mag einem die zuletzt genannte Position menschlich und moralisch sympathischer erscheinen, so enthält sie doch ein potentielles Problem für eine politische Gemeinschaft. Ist kulturelle Heterogenität grundsätzlich zu befürworten und ein Grundstein demokratischer Werte, so gilt doch gleichzeitig die Notwendigkeit eines die Gesellschaft zusammenhaltenden gemeinsamen Elementes auf der Ebene der politischen Kultur. Anders gesagt: Für die Persistenz eines politischen Systems benötigt man - folgt man der oben

38 Werner Schiffauer, Parallelgesellschaften. Wie viel Wertekonsens braucht unsere Gesellschaft? Für eine kluge Politik der Differenz, Bielefeld 2008.

ZfP 61. Jg. 2/2014 
vorgestellten politischen Kulturforschung - zumindest auf dem Sektor der politischen Unterstützung der Institutionen und Autoritäten und insbesondere der Verfassung einen gewissen Konsens. Wichtig ist, dieser ist auf der politischen Ebene angesiedelt. Entsprechend gibt nicht die Bestimmung kultureller Differenzen Auskunft über die Integrationsbereitschaft auf der politischen Ebene, sondern allein die Frage danach, ob es so etwas wie einen Konsens über die Legitimität der Demokratie und ihrer Werte in allen Teilen der religiös pluralen Bevölkerung gibt. ${ }^{39}$

Betrachten wir das erste Argument. Inwieweit sind die Muslime nicht bereit sich in die deutsche politische Kultur zu integrieren? Hier ließen im März 2012 getätigte Äußerungen des damaligen Innenministers Friedrich aufhorchen, der eine teilweise hohe Integrationsunwilligkeit junger Muslime in Deutschland ausmachte. Bei näherem Hinsehen begründeten sich diese Aussagen allerdings auf 20-25\% einer Gruppe von jungen deutschen Muslimen zwischen 14 und 32. Geht man davon aus, dass dann drei Viertel dieser Gruppe sich selbst als integrationsbereit einstufen, dann ist dies eigentlich kein besonders ungünstiges Ergebnis. ${ }^{40}$ Und die Betrachtung der Daten des Bertelsmann Religionsmonitors 2013 stärkt diese Annahme: So ist die Zustimmung zur Demokratie als guter Regierungsform - nach Überlegungen des Konzepts der politischen Kulturforschung als Legitimität der Demokratie zu verstehen ${ }^{41}$ - bei den Muslimen in Westdeutschland kaum geringer als bei ihren christlichen oder konfessionslosen Nachbarn (Abb. 4). Aufgrund der geringen Fallgröße macht eine Betrachtung ostdeutscher Muslime wenig Sinn. Bemerkenswert ist der Befund, dass die ostdeutschen Konfessionslosen in der Demokratielegitimität sogar hinter den westdeutschen Muslimen zurückstehen. Vermutlich ist dies aus der noch immer bestehenden Verzahnung zwischen ostdeutscher Identität und Konfessionslosigkeit zu erklären. Ein Grund für die höheren Werte des muslimischen Bevölkerungsanteils könnte in der positiv wahrgenommenen Schutzfunktion der Demokratie für religiöse Minderheiten liegen.

39 Damit wird an dieser Stelle eine klare Ausrichtung auf politische Integration im Sinne der politischen Kulturforschung gelegt und Aspekte der ökonomischen und der kulturellen Integration werden zurückgestellt. Dies erscheint mit Blick auf die Frage nach Religionspolitik auch berechtigt.

40 Vor allem wenn man sich vor Augen führt, dass bei einem Einbezug älterer Muslime mit deutscher Staatsangehörigkeit die Bereitschaft zu Antisystemverhalten und Integrationsabwehr deutlich absinken dürfte. Vgl. Bundesministerium des Inneren, Lebenswelten junger Muslime in Deutschland, Berlin 2012.

41 Vgl. Fuchs, Das Konzept der politischen Kultur: Die Fortsetzung einer Kontroverse in konstruktiver Absicht, aaO. (FN 19). 


\section{Abb. 4: Demokratielegitimität und Demokratiezufriedenheit im Religionsvergleich}

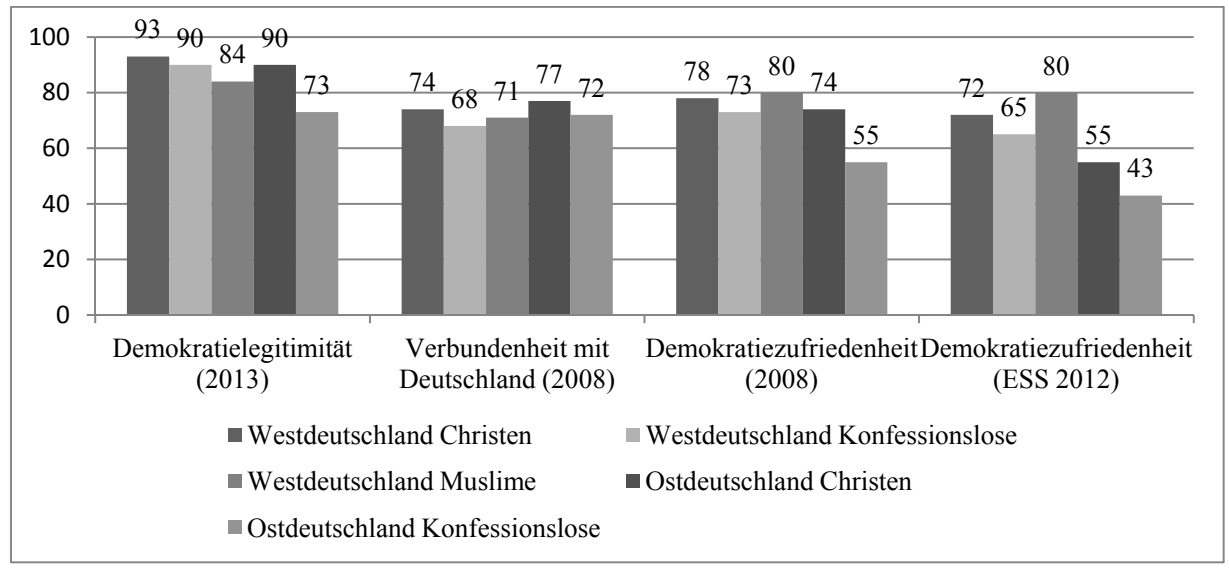

Quelle: Bertelsmann Religionsmonitor 2013: Demokratielegitimität $=$ Demokratie ist gute Regierungsform (Anteil befragter Muslime, n=322), Zustimmende Antworten (stimme voll und ganz zu + stimme eher zu) auf Vier-Punkte-Skala; Allbus 2008: Demokratiezufriedenheit, Verbundenheit mit Deutschland, Zustimmende Antworten auf Vier-Punkt-Skala (Anteil befragter Muslime n=96); European Social Survey 2012: Demokratiezufriedenheit, Zustimmende Antworten 6-10 auf einer Elf-Punkt-Skala (Anteil befragter Muslime n=72).

Und auch Aussagen zur Zufriedenheit mit der aktuellen Demokratie zeigen (aus dem Allbus 2008), dass aus einen Anstieg der Angehörigen islamischen Glaubens keine akute Gefahr für die politische Kultur zu resultieren scheint. Zwar mag es sein, dass die Muslime in Deutschland andere kulturelle Riten pflegen, sich sozial anders als Christen (oder Konfessionslose) organisieren und Religion einen höheren Stellenwert in ihrem Leben zuerkennen, als dies andere hier lebende Menschen tun, aber der Demokratie stehen die meisten von ihnen genauso positiv gegenüber wie ihre Mitbürger christlichen Glaubens. ${ }^{42}$

Insgesamt gesehen scheint eine vereinfachte Annahme eines Mangels an Zugewandtheit zur Demokratie auf die muslimische Bevölkerung in Deutschland anhand der meis-

42 Daraus ist keineswegs auf eine hohe Demokratiekonformität des Islam an sich oder entsprechende Haltungen in anderen Ländern zu schließen. Die Daten des Bertelsmann Religionsmonitors 2013 sowie der WZB-Studie "Six Country Immigrant Comparative Survey (SCIICS) « zeigen hier deutlich problematischere Beziehungen zwischen Demokratielegitimität (im Verständnis der Akzeptanz von Gesetzen) und einem nicht wenig verbreiteten islamischen Dogmatismus, wenn nicht gar Fundamentalismus auf. Gleichzeitig muss eine »fundamentalistische Position « nicht zwingend in Demokratieablehnung münden. Sie kann allerdings den Schwerpunkt auf die Ablehnung liberaler Wertvorstellungen legen, die man aus Sicht der Mehrheitsgesellschaft als eher einer Befürwortung wert ansieht. In dieser Hinsicht geben auch Zustimmungswerte von um die 30\% in der SCIICS-Studie zu fundamentalistischen Aussagen unter den deutschen Muslimen zu denken, in denen zum Beispiel ein Vorrang religiöser Regeln vor säkularen Gesetzen abgefragt wird. Ruud Koopmans, »Fundamentalismus und Fremdenfeindlichkeit. Muslime und Christen im europäischen Vergleich« in: WZB-Mitteilungen 142, 2013, S. 21-25. 
ten der hier präsentierten Daten nicht zuzutreffen. Dieser Befund ist auch plausibel. So setzen die nach Deutschland eingewanderten Muslime - und deren Kinder und Enkel sowieso - vermutlich auf den in Demokratien gesicherten Schutz von Minderheiten. Als Mitglied einer Minderheit erscheint eine solche rechtlich gewährte Sicherheit vorteilhaft. Zudem darf auch nicht unterschätzt werden, dass viele Muslime die individuellen Freiheiten, die ihnen die Demokratie gewährt, wohl als positiv empfinden.

Anders sieht dies aus, wenn man den Blick von der muslimischen Minderheit auf die Mebrheitsgesellschaft lenkt. Aus den Zahlen der 2010 durchgeführten Münsteraner Studie »Wahrnehmung und Akzeptanz religiöser Vielfalt« (Tab. 2) wird sichtbar, die Ablehnung der deutschen Mehrheitsgesellschaft konzentriert sich vor allem auf eine spezifische religiöse Gruppe, nämlich die Muslime und den Islam. Andere religiöse Gruppen lenken erheblich seltener negative Haltungen auf sich. So ist die Haltung den Hindus und den Buddhisten gegenüber in der deutschen Bevölkerung mehrheitlich positiv geprägt. Dies gilt auch für Deutsche jüdischen Glaubens. Sicherlich, es finden sich auch für die zuletzt genannten Gruppen noch - teils beachtliche - Minderheiten mit negativen Haltungen in West- wie in Ostdeutschland, breite Mehrheiten wie bei den Muslimen aber eben doch nicht. ${ }^{43}$

Tab. 2: Persönliche Haltung zu Mitgliedern anderer religiöser Gruppen

\begin{tabular}{lcccccccccccc}
\hline & \multicolumn{2}{c}{ Muslime } & \multicolumn{2}{c}{ Christen } & \multicolumn{2}{c}{ Hindus } & \multicolumn{2}{c}{ Buddhisten } & \multicolumn{3}{c}{ Juden } & \multicolumn{3}{c}{ Atheisten } \\
& W & $\mathrm{O}$ & $\mathrm{W}$ & $\mathrm{O}$ & $\mathrm{W}$ & $\mathrm{O}$ & $\mathrm{W}$ & $\mathrm{O}$ & $\mathrm{W}$ & $\mathrm{O}$ & $\mathrm{W}$ & $\mathrm{O}$ \\
\hline Sehr positiv & 6 & 3 & 35 & 23 & 5 & 4 & 13 & 8 & 8 & 6 & 11 & 28 \\
Eher positiv & 28 & 23 & 55 & 56 & 45 & 33 & 52 & 42 & 50 & 48 & 43 & 46 \\
\hline Eher negativ & 41 & 40 & 6 & 11 & 20 & 21 & 14 & 19 & 23 & 20 & 22 & 10 \\
Sehr negativ & 17 & 22 & 1 & 4 & 5 & 10 & 4 & 8 & 5 & 10 & 8 & 4 \\
\hline Weiß nicht & 5 & 9 & 1 & 4 & 20 & 27 & 13 & 19 & 9 & 13 & 11 & 9 \\
\hline
\end{tabular}

Quelle: Restwerte auf 100\% entsteht aus Angabe »keine Antwort «; W Westdeutschland, $\mathrm{O}=$ Ostdeutschland. ${ }^{44}$

Woran liegt diese ablehnende Haltung gerade einer spezifischen religiösen Gruppe gegenüber? Man liegt wohl nicht falsch, wenn man hier auf ein durch verschiedene Wahrnehmungsmechanismen ausgebildetes Stereotyp schließt. So kann es gerade in den neuen Bundesländern, wo die Zahl der Muslime ja sehr gering ist, nicht an persönlichen Erfahrungen liegen, dass man Mitgliedern dieser speziellen sozialen Gruppe mehrheitlich negativ gegenübersteht. Die Haltungen müssen auf anderem Wege als durch Kontakte ent-

43 Alexander Yendell, »Muslime unerwünscht? Zur Akzeptanz des Islam und dessen Angehörigen. Ein Vergleich zwischen Ost- und Westdeutschland « in: Gert Pickel / Oliver Hidalgo (Hg.), Religion und Politik im vereinigten Deutschland. Was bleibt von der Rückkehr des Religiösen?, Wiesbaden 2013, S. 221-248.

44 Vgl. Yendell, »Muslime unerwünscht? Zur Akzeptanz des Islam und dessen Angehörigen. Ein Vergleich zwischen Ost- und Westdeutschland«, aaO. (FN 43), S. 232; Pollack u.a., Grenzen der Toleranz, aaO. (FN 4), S. 20, 51-53, 64-65. 
stehen. Ein zentrales Erklärungsmotiv stellen Bedrohungswahrnehmungen dar. So ist aus der Stereotypenforschung bekannt, dass speziell die Wahrnehmung einer Bedrohung durch eine andere Gruppe eine Ablehnung dieser hervorruft. ${ }^{45}$ Genau dies trifft für die Haltung zu den Muslimen zu. Spätestens seit dem 11. September 2001 wird der Islam von vielen Menschen als bedrohlich erachtet. Dies zeigt auch Abbildung 5. Erneut treten die Differenzen in der Beurteilung der verschiedenen Religionen hervor und sie entsprechen im Vergleich der Religionen den bereits oben dargestellten Haltungen. So überschreitet nur beim Islam der Anteil derjenigen, die religiöse Pluralisierung als Bedrohung erachten, den Anteil derjenigen, die diesen Aspekt als Bereicherung einschätzen. Alle anderen Religionen werden häufiger als Bereicherung denn als Bedrohung wahrgenommen.

\section{Abb. 5: Wabrnehmung religiöser Pluralisierung in Deutschland}

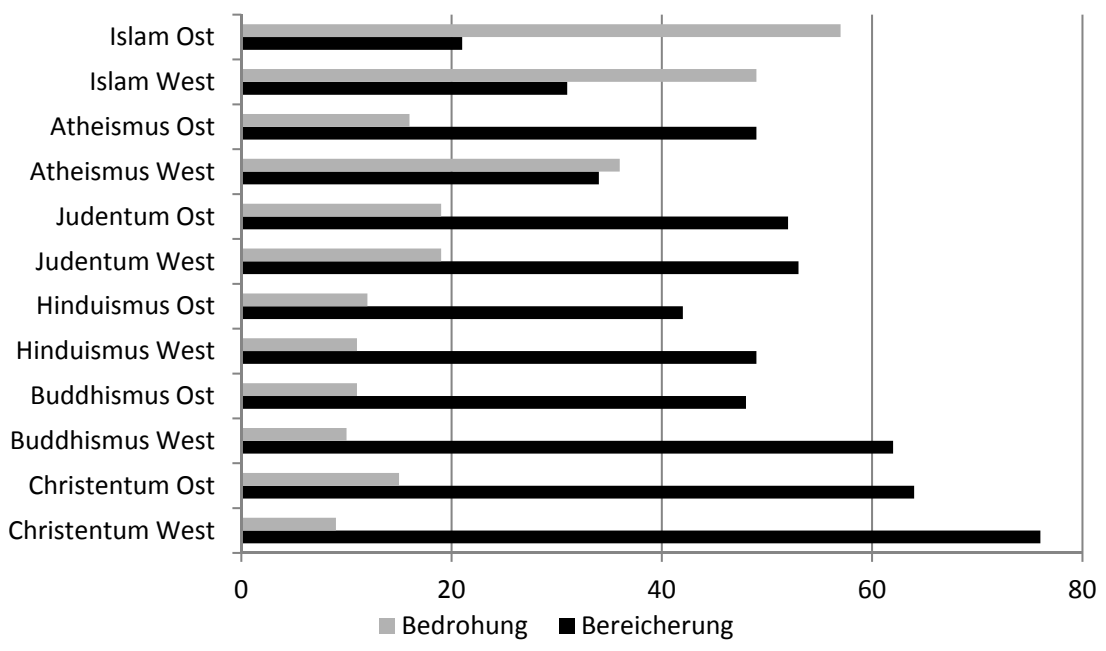

Quelle: Eigene Berechnungen, Bertelsmann Religionsmonitor 2013; Wenn Sie an die Religionen denken, die es auf der Welt gibt: Als wie bedroblich bzw. wie bereichernd nehmen sie die folgenden Religionen wabr? Anteile: sehr bedroblich/eher bedroblich; sebr bereichernd/bereichernd.

In den letzten beiden Abbildungen zeigt sich noch ein weiterer Befund: Zum einen ist in Westdeutschland die Skepsis gegenüber dem Atheismus immer noch etwas stärker ausgeprägt als in Ostdeutschland, zum anderen wird in Ostdeutschland etwas häufiger das Christentum als bedrohlich identifiziert. Diese Ergebnisse sind aufgrund der unterschiedlichen religiösen Kulturen in West- und Ostdeutschland nicht unbedingt verwunderlich. Bemerkenswert ist eher die übersichtliche Größe der Unterschiede. So scheint

45 Vgl. Immo Fritsche / Eva Jonas / Thomas Kessler, "Collective Reactions to Threat: Implications for Intergroup Conflict and for Solving Societal Crisis « in: Social Issues and Policy Review 5/1 (2011), S. 101-136. 
sich die Situation zwischen den westdeutschen Christen und ostdeutschen Konfessionslosen immer mehr zu entspannen und eine auf religiösen oder nichtreligiösen Aspekten beruhende wechselseitige Ablehnung aufzulösen. Für das Gros der deutschen Bevölkerung spielen Zugehörigkeit zum Christentum oder deren Fehlen nur mehr eine sehr nachgeordnete Rolle für das gemeinsame Alltagsleben. Bedrohungswahrnehmungen wie zum Beispiel in den USA, wo von einer Mehrheit der Bevölkerung der Atheismus als konkrete Bedrohung ausgemacht wird, findet man in Deutschland nicht. ${ }^{46}$

Diese Entspannung hat auch Konsequenzen für den politischen Umgang mit Forderungen des neuen Atheismus. Die Forderungen des organisierten Atheismus scheinen nämlich nur auf eine begrenzte Legitimität in der Bevölkerung zurückgreifen zu können. Zum einen stehen kaum breite Bevölkerungsgruppen aktiv hinter der antireligiösen Politik dieser Organisationen, zum anderen sieht die Mehrheit der Deutschen keinen wesentlichen Konfliktstoff an dieser Stelle. Zwar empfinden viele Westdeutsche die »Kultur der Konfessionslosigkeit « im Osten Deutschlands als fremd und können diese nicht nachvollziehen, eine zentrale Bedeutung für ihre politischen Entscheidungen und das Zusammenleben in einer politischen Gemeinschaft besitzt dies allerdings nicht. Das ist auch vor dem Hintergrund einer aus Sicht fast aller Bürger weitgehend säkular verfassten Gesellschaft zu sehen, die auf weitgehend akzeptierten Prozessen funktionaler Differenzierung beruht. Wenn Religion eher im privaten Bereich angesiedelt wird und Vertreter religiöser Gruppierungen sich von der Politik fernhalten sollten, dann fehlt die Grundlage für einen diesbezüglichen Konflikt - und auch die Vertreter eines (teils ideologisch scheinenden) Atheismus sollten sich aus Sicht der Bürger aus der Politik heraushalten. Die Akzeptanz einer weit reichenden gesellschaftlichen Säkularität durch die deutschen Bürger bedeutet dementsprechend bei weitem keine Zuwendung zum politischen Atheismus. ${ }^{47}$

Anders ist die Situation hinsichtlich des Islam. Hier besteht zwischen Westdeutschen und Ostdeutschen eine bemerkenswerte Einigkeit in der skeptischen bis ablebnenden Haltung. Im Prinzip ist die Bezeichnung der entsprechenden öffentlichen Kontroversen als Integrationsdebatte eher ein Euphemismus denn eine Realitätsbeschreibung. In Wirklichkeit handelt es sich wohl eher um eine dezidierte Islamdebatte. Nicht nur, dass in West- wie in Ostdeutschland ungefähr drei von fünf Befragten eine negative Haltung gegenüber Muslimen bekennen; über die Hälfte der Bürger in beiden Gebieten fühlt sich auch durch den Islam bedroht (Tab. 2, Abb. 5). Nach Daten des Allbus 2010 sehen hier auch $60 \%$ der West- wie der Ostdeutschen für die Zukunft ein zentrales Konfliktfeld in Deutschland. In diesen Äußerungen kommen Bedrohungswahrnehmungen, kulturelle Fremdheit und die Angst vor dem Fremden zusammen. Dass man dabei in den Medien den Islam relativ konsistent als gewalttätig und feindlich präsentiert bekommt, tut sein

46 Vgl. Gert Pickel, Bertelsmann Religionsmonitor 2013. Religiosität im internationalen Vergleich, Gütersloh 2013, S. 29.

47 Gladkich / Pickel, »Politischer Atheismus. Der neue Atheismus als politisches Projekt oder Abbild empirischer Realität?«, aaO. (FN 6), S. 125-148. 
übriges um die entsprechenden Stereotype auszubilden. ${ }^{48}$ Sie lassen den Islam in den Augen der deutschen Bürger mehrheitlich als gewalttätig, fanatisch und vor allem frauenfeindlich erscheinen. ${ }^{49}$

Tab. 3: Konsequenzen der Haltung zu Muslimen

\begin{tabular}{|c|c|c|c|c|}
\hline & \multicolumn{2}{|c|}{ Westdeutschland } & \multicolumn{2}{|c|}{ Ostdeutschland } \\
\hline & $\mathrm{Ja}$ & Nein & $\mathrm{Ja}$ & Nein \\
\hline Befürwortung Moscheebau (2010) & 28 & 65 & 19 & 74 \\
\hline Befürwortung Minarettbau (2010) & 18 & 75 & 12 & 80 \\
\hline \multirow{2}{*}{$\begin{array}{l}\text { Befürwortung muslimischer Feier- } \\
\text { tag (2010) }\end{array}$} & 17 & 77 & 13 & 81 \\
\hline & $\begin{array}{l}\text { Zustim- } \\
\text { mung }\end{array}$ & Ablehnung & $\begin{array}{l}\text { Zustim- } \\
\text { mung }\end{array}$ & Ablehnung \\
\hline $\begin{array}{l}\text { Zunehmende Anzahl der Muslime } \\
\text { als Konfliktursache (WArV 2010) }\end{array}$ & 72 & 25 & 77 & 22 \\
\hline $\begin{array}{l}\text { Wünschen ein Limit der Zuwan- } \\
\text { derung für Muslime (WArV 2010) }\end{array}$ & 65 & 30 & 76 & 18 \\
\hline $\begin{array}{l}\text { Einschränkung islamischer Glau- } \\
\text { benspraktiken (WArV 2010) }\end{array}$ & 42 & 52 & 55 & 40 \\
\hline $\begin{array}{l}\text { Islam passt in die deutsche Gesell- } \\
\text { schaft (Allbus 2012) }\end{array}$ & 8 & 46 & 6 & 59 \\
\hline $\begin{array}{l}\text { Muslimische Bürgermeister sind in } \\
\text { Ordnung (Allbus 2012) }\end{array}$ & 27 & 40 & 21 & 50 \\
\hline $\begin{array}{l}\text { Man sollte Islamausübung be- } \\
\text { schränken (Allbus 2012) }\end{array}$ & 19 & 43 & 31 & 29 \\
\hline $\begin{array}{l}\text { Staat sollte islamische Gruppen be- } \\
\text { obachten (Allbus 2012) }\end{array}$ & 30 & 25 & 41 & 16 \\
\hline
\end{tabular}

Quelle: WArV 2010, Allbus 2012; Zunehmende Anzabl der Muslime als Konfliktursache = Zustimmung = stimme stark $\mathrm{z} u+$ stimme eher $\mathrm{zu}$; Ablebnung = stimme eher nicht $\mathrm{z} u+$ stimme überhaupt nicht zu; Residuale Werte sind weiß nicht oder keine Angabe (bei Allbus 2012 zusätzlich Mittelkategorie stimme weder zu noch lebne ab).

48 Dies bezieht sich auf die eher diffuse, deswegen aber umso wirkungsmächtigere Berichterstattung über Krisengebiete, in denen der Islam und Islamisten in der Regel eine negative Darstellung erfahren. Liberale oder offenere Tendenzen im Islam sind den meisten deutschen (wie auch europäischen) Bürgern weitgehend unbekannt bzw. nehmen kaum Raum in der Medienberichterstattung ein. Vgl. hierzu auch Katajun Amirpur, Den Islam neu denken: Dschibad für Demokratie, Freibeit und Franenrechte, München 2013.

49 Vgl. Pollack u.a., Grenzen der Toleranz, aaO. (FN 4), S. 21-23; siehe generell auch die Erkenntnisse der Stereotypenforschung bei Gordon W. Allport, The Nature of Prejudice, Cambridge, 1954; Henri Tajfel, Gruppenkonflikt und Vorurteil. Entstehung und Funktion sozialer Stereotypen, Bern, 1982. 
Da ist es dann kein Wunder, wenn bei der Frage, ob die zunehmende Zahl an Muslimen als Konfliktursache angesehen werden muss, drei Viertel der Bürger in West- und in Ostdeutschland dieser Aussage eher oder gar stark zustimmen. Bedrohungswahrnehmungen und eine daraus resultierende Furcht vor Konflikten sind eng miteinander verbunden. ${ }^{50}$ Der Islam wird entsprechend als Religion angesehen, die mehrheitlich nicht in die westliche, und damit auch nicht in die deutsche Gesellschaft passt. Auch eine Reihe anderer Indikatoren zeichnet dieses Bild, welches ganz erhebliche Konsequenzen auf der politischen Entscheidungsebene mit sich bringt. So wären, würden die Bürger direkt darüber abstimmen, sowohl Bestrebungen eines Baus von Moscheen als auch von Minaretten ein relativ hoffnungsloses Unterfangen. Auch hier sind es drei Viertel der Deutschen, welche beides ablehnen. Ganz zu schweigen von der Einführung eines muslimischen Feiertags, der nicht einmal für ein Fünftel der Deutschen eine auf Zustimmung treffende Option darstellt. ${ }^{51}$

Aus diesen Haltungen entstehen erhebliche Forderungen (demands), die an die Politik gestellt werden. So findet sich eine klare Mehrheit, welche Begrenzungen für Zuwanderung von Muslimen fordert (Tab. 3). Hinter solchen Äußerungen stecken erhebliche Ängste vor Gefahren der »Überfremdung « und wahrgenommener kultureller Bedrohung. Sie sind von konkreten Gefährdungslagen unabhängig. Zwar würde eine größere Minderheitengruppe, die eine fundamentalistische Haltung einnimmt, die Konfliktlage verschärfen, für die Ablehnung seitens der Mehrheitsgesellschaft und deren politischen Folgen ist sie nicht notwendig. Diese Ängste können dementsprechend weitreichende mittelbare Effekte erzielen, richten sich doch die Forderungen an Parteien und besteht doch bei ihrer Nichterfüllung die Gefahr eines Unterstützungsentzugs und eines Entzugs von Wählerstimmen. Gleichzeitig könnten diese Einstellungen aber langfristig auch über Forderungen nach spezifischer Unterstützung hinausgehen und eine grundsätzlichere Frage an die deutsche Demokratie stellen - und damit die Legitimität der deutschen Demokratie insgesamt beschädigen. Davon ganz abgesehen, kann man auch einmal darüber nachdenken, welche Konsequenzen bei in dieser Richtung zielenden Volksbegehren und Volksentscheiden zu erwarten sind. Klar ist: Es ist weniger die religiöse Pluralisierung an sich, die Probleme mit sich bringt. Vielmehr sind es die Folgen in den Köpfen der Bürger, welche das politische System der Bundesrepublik vor Herausforderungen stellen. ${ }^{52}$

50 So kommen Pollack und Kollegen in ihrer Fünfländerstudie sogar zu dem Befund, dass die Einschätzung religiöser Pluralisierung als konfliktär in Deutschland als einzigem Vergleichsland stärker ist als die Wahrnehmung als Bereicherung. Vgl. Pollack u.a., Grenzen der Toleranz, aaO. (FN 4), S. 18; auch Pickel, Bertelsmann Religionsmonitor 2013. Religiosität im internationalen Vergleich, aaO., (FN 46), S. 33-34.

51 Michael Blohm / Martina Wasmer, »Einstellungen und Kontakte zu Ausländern « in: Statistisches Bundesamt (Hg.), Datenreport 2013. Ein Sozialbericht für die Bundesrepublik Deutschland, Bonn 2013, S. 205-211.

52 Gert Pickel, »Bedrohungsgefühle versus vertrauensbildende Kontakte - Religiöser Pluralismus, religiöses Sozialkapital und soziokulturelle Integration «. in: Detlef Pollack / Ingrid Tucci / Hans-Georg Ziebertz (Hg.), Religiöser Pluralismus im Fokus quantitativer Religionsforschung, Wiesbaden 2012, S. 221-264. 


\section{Fazit - Politische Herausforderungen durch religiösen Wandel}

Es dürfte unstrittig sein, dass in den letzten Jahrzehnten ein massiver Wandel des Religiösen stattgefunden hat. Prozesse der individuellen und gesellschaftlichen Säkularisierung finden parallel zu einer kontinuierlich voranschreitenden religiösen Pluralisierung statt. Die Zahl der Konfessionslosen steigt an, und zugleich nimmt auch die Zahl der Mitglieder anderer Religionsgemeinschaften zu, die nicht (mehr) einer der beiden jahrhundertelang dominierenden Konfessionen, d.h. dem Katholizismus oder dem Protestantismus, angehören. Speziell die Mitglieder muslimischer Glaubensgemeinschaften und orthodoxe Christen legen in ihrem Anteil an der deutschen Bevölkerung prozentual stetig zu. Dies verändert die Sicht auf die religiöse Situation in Deutschland, muss die Gesellschaft doch, bei allem immer noch bestehenden Übergewicht der beiden großen christlichen Konfessionen, verstärkt als multireligiöse Gesellschaft gesehen werden.

Aus diesem religiösen Wandel resultieren verschiedene Herausforderungen für die deutsche Politik. ${ }^{53}$ Dabei handelt es sich generell weniger um die religiöse Pluralisierung als sozialstrukturelle Tatsache, sondern weit stärker um die Folgen, welche durch eine gestiegene Aufmerksamkeit für diese religiöse Pluralisierung in den Köpfen der Bürger der politischen Gemeinschaft entstehen. So könnte eine Zunahme der Konfessionslosen zwar theoretisch zu einer politisch-legitimatorischen Stärkung von Organisationen führen, die eher atheistische Positionen vertreten. Diese Zunahme ist aber für die politische Kultur in Deutschland weitgehend folgenlos geblieben. Anders ist es bei der Debatte um religiöse Pluralisierung. Diese mündete in den letzten Jahren in eine kontroverse und politisch brisante Debatte über den Islam sowie der politischen Ausrichtung seiner Anhänger. Folge ist seine (pauschale) Ablehnung durch eine beträchtliche Zahl der Bürger. Dabei ist es explizit der Islam und keine andere Religion, welcher mit den »Gefahren der religiösen Pluralisierung « in Verbindung gebracht wird.

Die letzte Entwicklung ist nun als eine Problemlage zu markieren, die langfristig den Zusammenhalt einer politischen Gemeinschaft gefährden könnte. Es etablieren sich Stereotype und Abgrenzungen innerhalb der politischen Gemeinschaft, welche ein konfliktfreies Zusammenleben zumindest erschweren. Dabei sind es in Deutschland aus diesem Blickwinkel scheinbar weniger die Muslime, welche im Blick auf die Veränderung der politischen Kultur Zielpolitischen Handelns sein müssten, als vielmehr die Masse der Bürger, die muslimische Gruppen derzeit skeptisch bis gar feindlich gegenübersteht. ${ }^{54}$ Folgt man kritischeren Sichten der Integrationsfähigkeit der Muslime ${ }^{55}$, dann verschärft sich das Problem politischer Konfliktgefahren sogar noch, da dann beide Seiten politische

53 Wobei dieser Anspruch gut zumindest auf die anderen Länder Westeuropas auszuweiten ist, die vor den gleichen Problemen und Herausforderungen stehen.

54 Ohne Frage ist ein Abbau von Fremdheit auf beiden Seiten, der Mehrheits- und der Minderheitsbevölkerung, einem Konfliktabbau förderlich. Für die politische Kultur zuerst einmal dringlicher ist es aber wohl das Bild vom Islam und die Haltungen Mitbürgern muslimischen Glaubens gegenüber positiv zu verändern.

55 Koopmans, »Fundamentalismus und Fremdenfeindlichkeit. Muslime und Christen im europäischen Vergleich« aaO (FN 42), S. 22-23. 
Handlungen herausfordern. Entscheidend ist aber, dass die religiöse Fremdheit ein sogar von dem realen Verhalten der anderen gruppe unabhängiges Problempotential produziert. Die grundsätzliche Bedeutung von Religion für die politische Gemeinschaft wird durch die zunehmende Auflösung der Differenzierung zwischen muslimischen Migranten und bereits über Generationen eingesessenen Muslimen verdeutlicht. So ist das Identifikationsmerkmal nicht mehr die Fremdheit der Zuwanderung, sondern die Fremdheit der anderen Religion (und Kultur).

Noch ist es nicht klar, ob diese sich ausbildenden Konfliktsituationen im Sinne einer übergreifenden Religionspolitik angegangen werden können - oder angegangen werden. Auch ist nicht klar, ob sie durch eine spezifische Religionspolitik gelöst werden können. Das Problem allein auf den Feldern von Integrations-, Ausländer- oder Innenpolitik zu lösen, erscheint vor diesem Hintergrund allerdings kaum möglich. Wie oben angesprochen treffen einfache Politiken im Zuwanderungsbereich bei weitem nicht mehr den Kern der Probleme, erfolgen die Gruppenzuweisungen doch anhand anderer Abgrenzungsmerkmale. Wesentlich sinnvoller erscheint es, eine Religionspolitik, welche die Spezifika unterschiedlicher Religionen deutlich und dem Bürger verständlich macht, zwischen den politischen Handlungsträgern zu koordinieren und mit den ebenfalls beteiligten PolicyFeldern der Migrations-, Moral- und Identitätspolitik zu verknüpfen. Dass dies oft parteienübergreifend geschieht, zeigen schon einige der bislang erfolgten Diskussionen über religiöse Themen und Zugehörigkeiten. Dies ist positiv zu bewerten, handelt es sich doch bei der Religionspolitik um eine die Parteipolitik übersteigende Aufgabe der deutschen (europäischen) Politik und politischen Kultur. Nur auf diese parteipolitisch integrative Weise kann es gelingen, die aus diesen Problemlagen entstehenden Gefährdungsszenarien der deutschen politischen Kultur anzugehen.

Und Religionspolitik benötigt man auch unter den Bedingungen einer zunehmenden individuellen und gesellschaftlichen Säkularisierung. So bedeutet es nicht, dass mit einer sinkenden Zahl an religiösen Menschen Debatten und Konflikte über Religion an Bedeutung verlieren. Es kann sogar sein, dass diese aufgrund von stärkerer Polarisierung in der Gesellschaft an Bedeutung gewinnen. Entsprechend braucht es auch keine Wiederkehr der Religionen oder des Religiösen, für die es ja auch nur wenig Anzeichen gibt. Ein Bedeutungszuwachs von Religion auf der politischen Ebene kann sehr wohl Hand in Hand gehen mit individueller Säkularisierung, wenn er nicht sogar durch diese ausgelöst wird. ${ }^{56}$

\section{Zusammenfassung}

Deutschland wie auch seine europäischen Nachbarstaaten wurden in den letzten Jahrzehnten von einem tiefgreifenden Wandel des Religiösen betroffen. Neben der Ausbrei-

56 Zudem kann man darauf verweisen, dass wir es selbst in Europa ja keineswegs mit breitflächig säkularen Gesellschaften auf der individuellen Ebene zu tun haben. Denn ist es nicht so, dass es noch immer übergreifende religiös-kulturelle Verständnisse sind, die die europäischen Gesellschaften prägen? Säkularisierung bedeutet ja auch nicht Säkularität. 
tung der Konfessionslosigkeit in Westeuropa sind es vor allem Prozesse der religiösen Pluralisierung, die in immer stärkerem Umfang Raum greifen. Speziell die Zunahme der Bevölkerung muslimischen Glaubens kennzeichnet diese Prozesse. Mit diesem religiösen Wandel sind aber auch Herausforderungen der politischen Kultur in Deutschland verbunden. So stellt sich gerade für die Ebene der politischen Gemeinschaft die Frage, ob die in der Integrationsdebatte thematisierten Konflikte nicht zu einer Zerreißprobe der deutschen politischen Kultur führen können. Empirische Ergebnisse zeigen dabei, dass speziell die Haltung der deutschen Bürger gegenüber Muslimen »Integrationsprobleme « der gesamten politischen Gemeinschaft mit sich bringen können. Um diesen Problemen entgegenzutreten, könnte eine mit anderen Politikfeldern verknüpfte Religionspolitik möglicherweise Antworten bereitstellen.

\section{Summary}

Germany and its European neighbours are facing a far reaching change of their religious landscapes during the last decades. This religious change is characterized by two major processes: the increasing number of non-denominationals on the one hand and the continuing pluralization of the religious field on the other. Both developments affect the German political culture and create new challenges. It is to be emphasized that especially the expansion of the religious spectrum by an increasing number of Muslims could lead to a crucial test for the German political community. Reasons for that are not, as often alleged, the unwillingness to integrate on the part of the immigrants but a quite skeptical or even negative attitude of German society towards Muslim citizens as it is manifested by empirical survey data. An alliance between the politics of religion and other areas of polities like integration, moral or identity could provide answers or new perspectives to encounter these problems, that may threaten the political community of Germany.

Gert Pickel, Religious Change as a Challenge for Germany's Political Culture-Religious Pluralization and Secularization as Catalyst of a (new) Politics of Religion? 